\title{
Disturbance Compensating Adaptive Regulator for MIMO Time-Delayed Discrete-Time Systems
}

\author{
Khalid ABIDI \\ Electrical Power Engineering Programme \\ Newcastle University Singapore \\ Singapore \\ khalid.abidi@ncl.ac.uk
}

\author{
Hang Jian SOO \\ Not Affiliated \\ Singapore \\ radagast@ieee.org
}

\begin{abstract}
A discrete-time adaptive regulator for uncertain MIMO LTI systems with input delays is proposed. The input delays are assumed to be constant and unknown except for a known upper bound, and it is possible for delays to be different across input channels. An adaptive estimator is used for online parameter estimation, and the control law is obtained by applying Artstein's model reduction to the adaptive model after is has been rewritten using second order difference. The resulting controller is, then, able to stabilize the system while mitigating the effects of the unmeasurable exogeneous disturbances.
\end{abstract}

Index Terms-Delay Systems, Discrete-time Systems, Adaptive Control

\section{INTRODUCTION}

Systems with time delays are found in many industrial processes [1] [2], and these delays can cause instability in closed-loop control. It is sometimes possible for a controller that does not perform any explicit time delay compensation to stabilise a plant with time delay. Thus one direction of research that has been pursued is to determine the maximum time delay (i.e. delay margin) that a certain type of controller can deal with on a particular class of plant dynamics. A recent example is the study of the delay margins of PID controllers on $1 \mathrm{st} / 2 \mathrm{nd}$ order LTI systems [3].

For long time delays, it is necessary to identify the delay (for example, using the method in [4]) and compensate for it. Artstein's model reduction [5] is a popular time delay compensation method which has been adapted to the discretetime case in [6]. If the time delay is uncertain and it is not feasible to identify it (for example, because the same control software is to be deployed on mass-produced hardware with loose tolerances), then adaptive control may be an appropriate solution.

This paper proposes a discrete-time adaptive regulator for uncertain LTI plants with input time delays, subject to unmeasurable disturbances. It is based on previous work by the authors, where Artstein's reduction was applied as part of an adaptive controller for an uncertain scalar LTI plant [7]. This was later extended to the $\mathrm{n}^{\text {th }}$ order SISO case with disturbance in [8] (in press). The present work further extends this to the MIMO case, and moreover, addresses the possibility that in a

This work is supported by Newcastle University through the Research Support Allowance. multi-input plant the time delays in each input channel may not all be the same. Moreover, the second order difference and a deadzone in the parameter adaptation law are used to mitigate the effect of unmeasurable exogeneous disturbances.

\section{Problem Definition}

Consider the $n^{\text {th }}$ order MIMO system in continuous-time with input delay given as

$$
\dot{\mathbf{x}}(t)=A \mathbf{x}(t)+B \boldsymbol{\xi}(\mathbf{u})+\boldsymbol{\delta}(t)
$$

where the state vector is $\mathbf{x}(t) \in \mathbb{R}^{n}$ and the input vector is $\mathbf{u}(t) \in \mathbb{R}^{m}$. The scalar components of $\mathbf{u}(t)$ are denoted $u_{j} \in$ $\mathbb{R}$ for $j=1, \ldots, m$. Each input $u_{j}$ is subject to an uncertain, constant input time delay $\tau_{j} \in \mathbb{R}^{+}$. The delayed inputs vector

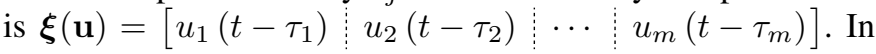
general, it is possible that certain delays are identical in duration while others differ, so that there may be only $q \leq m$ distinct delays. These will be denoted $\tau_{1}^{\prime}, \ldots, \tau_{q}^{\prime}$, such that $\tau_{1}^{\prime}<\tau_{2}^{\prime}<\cdots<\tau_{q}^{\prime}$. The matrices $A \in \mathbb{R}^{n \times n}$ and $B \in \mathbb{R}^{n \times m}$ are constant uncertain matrices and $\boldsymbol{\delta}(t) \in \mathbb{R}^{n}$ is an unmeasurable bounded exogenous disturbance.

Sampling the system (1) at a uniform time interval $T$ (where in general the time delays $\tau_{j} \forall j \in[1, m]$ may not be an integer multiple of $T$ ) yields the sampled-data model given by

$$
\mathbf{x}_{k+1}=F \mathbf{x}_{k}+\sum_{j=1}^{q}\left(G_{1, j} \mathbf{u}_{k-d_{j}}+G_{2, j} \mathbf{u}_{k-d_{j}-1}\right)+\boldsymbol{\omega}_{k}
$$

where $k \in \mathbb{Z}^{+}$corresponds to the $k^{\text {th }}$ time-step and $d_{1}, \ldots, d_{q} \in \mathbb{Z}^{+}$are the $q$ distinct, uncertain constant delays in time-steps that satisfy $d_{1}<d_{2}<\cdots<d_{q}$ and $d_{j} T \leq \tau_{j}^{\prime} \leq\left(d_{j}+1\right) T \quad \forall j \in[1, q]$. The matrices $F \in \mathbb{R}^{n \times n}$ and $G_{1, j}, G_{2, j} \in \mathbb{R}^{n \times m} \forall j \in[1, q]$ are computed as follows:

$$
\begin{aligned}
F & =e^{A T} \\
G_{1, j} & =\int_{0}^{\left(d_{j}+1\right) T-\tau_{j}^{\prime}} e^{A \sigma} d \sigma B_{j} \quad G_{2, j}=\int_{\left(d_{j}+1\right) T-\tau_{j}^{\prime}}^{T} e^{A \sigma} d \sigma B_{j}
\end{aligned}
$$

where $B_{j} \in \mathbb{R}^{n \times m}$ is obtained by taking the matrix $B$ and setting all entries to zero except those in columns corresponding 
to inputs delayed by $\tau_{j}^{\prime}$. The sampled disturbance $\boldsymbol{\omega}_{k} \in \mathbb{R}^{n}$ is given by

$$
\boldsymbol{\omega}_{k}=\int_{0}^{T} e^{A \sigma} B \boldsymbol{\delta}((k+1) T-\sigma) d \sigma
$$

The system (1) and the sampled-data system (2) satisfy the following assumptions:

Assumption 1: The disturbance $\boldsymbol{\delta}(t)$ is smooth and bounded and as a result $\left\|\boldsymbol{\omega}_{k}\right\| \leq \omega_{\max } \in O(T)$ and $\left\|\Delta^{2} \boldsymbol{\omega}_{k}\right\|=\| \boldsymbol{\omega}_{k}-$ $2 \boldsymbol{\omega}_{k-1}+\boldsymbol{\omega}_{k-2}\|=\| \boldsymbol{\nu}_{k} \| \leq \nu_{\max } \in O\left(T^{3}\right)$, [9].

Assumption 2: The delay $\tau_{\max }=\max \left\{\tau_{1}^{\prime}, \ldots, \tau_{q}^{\prime}\right\}$ is bounded as $\tau_{\max } \leq \tau_{\mathrm{p}}$ and $\tau_{\mathrm{p}}$ satisfies $p T \leq \tau_{\mathrm{p}} \leq(p+1) T$ where $p$ is the upper-bound on the delay in time-steps.

\section{MAIN RESUlT}

\section{A. Adaptive Estimator}

The discrete time plant model (2) can be written in a way that does not explicitly depend on knowledge of the actual time delays, but only their upper bound $p$ :

$$
\mathbf{x}_{k+1}=F \mathbf{x}_{k}+\sum_{i=0}^{p_{\mathrm{m}}} \Psi_{i} \mathbf{u}_{k-i}+\boldsymbol{\omega}_{k}
$$

where $p_{\mathrm{m}}=p+1$ and the parameters $\Psi_{i} \in \mathbb{R}^{n \times m}$ are defined appropriately so that the expression is equivalent to (2). Thus $\Psi_{i}$ may either be equal to $G_{1, j}, G_{2, j}, G_{2, j}+G_{1, j+1}$, or the zero matrix. It is also convenient to write this as

$$
\mathbf{x}_{k+1}=\Theta^{\top} \boldsymbol{\zeta}_{k}
$$

where $\Theta \triangleq\left[\begin{array}{llll}F & \Psi_{0} & \ldots & \Psi_{p_{m}}\end{array}\right] \in \mathbb{R}^{n \times\left[n+m\left(p_{\mathrm{m}}+1\right)\right]}$ and $\left.\boldsymbol{\zeta}_{k} \triangleq\left[\begin{array}{llll}\mathbf{x}_{k} & \mathbf{u}_{k} & \mathbf{u}_{k-1} & \ldots \mathbf{u}_{k-p_{\mathrm{m}}}\end{array}\right] \in \mathbb{R}^{\left[n+m\left(p_{\mathrm{m}}+1\right)\right.}\right]$

In order to estimate the uncertain parameters, define an adaptive plant model given by

$$
\begin{aligned}
\hat{\mathbf{x}}_{k+1} & =\hat{F}_{k} \mathbf{x}_{k}+\sum_{i=0}^{p_{\mathrm{m}}} \hat{\Psi}_{i, k} \mathbf{u}_{k-i} \\
& =\hat{\Theta}_{k}^{\top} \boldsymbol{\zeta}_{k}
\end{aligned}
$$

where $\hat{\Theta}_{k} \triangleq\left[\begin{array}{llll}\hat{F}_{k} & \hat{\Psi}_{0, k} & \ldots & \hat{\Psi}_{p_{\mathrm{m}}, k}\end{array}\right]$.

Define the model estimation error $\tilde{\mathbf{x}}_{k} \triangleq \mathbf{x}_{k}-\hat{\mathbf{x}}_{k}$, which has the dynamics

$$
\tilde{\mathbf{x}}_{k+1}=\tilde{\Theta}_{k} \boldsymbol{\zeta}_{k}+\boldsymbol{\omega}_{k}
$$

where $\tilde{\Theta}_{k} \triangleq \Theta-\hat{\Theta}_{k}$ is the parameter estimation error .

The adaptation laws used to obtain the parameter estimates $\hat{\Theta}_{k}$ are

$$
\begin{array}{r}
\hat{\Theta}_{k+1}= \begin{cases}\hat{\Theta}_{k}+\alpha_{k} \rho_{k} P_{k+1} \boldsymbol{\zeta}_{k} \tilde{\mathbf{x}}_{k+1}^{\top} & \forall k \in\left[k_{0}, \infty\right) \\
\hat{\Theta}_{k_{0}} & \forall k \in\left[0, k_{0}\right)\end{cases} \\
P_{k+1}= \begin{cases}P_{k}-\frac{\alpha_{k} \rho_{k} P_{\mathrm{x}, k+1}}{1+\alpha_{k} \rho_{k} \mu_{k}} & \forall k \in\left[k_{0}, \infty\right) \\
P_{k_{0}} & \forall k \in\left[0, k_{0}\right)\end{cases}
\end{array}
$$

$$
\rho_{k}= \begin{cases}1-\frac{\omega_{\max }}{\left\|\tilde{\mathbf{x}}_{k+1}\right\|} & \text { if }\left\|\tilde{\mathbf{x}}_{k+1}\right\| \geq \omega_{\max } \\ 0 & \text { if }\left\|\tilde{\mathbf{x}}_{k+1}\right\|<\omega_{\max }\end{cases}
$$

where $k_{0} \geq 0$ is the initial time-step, $\alpha_{k}>0$ is a positive coefficient that guarantees that $\left|\hat{F}_{k}\right| \neq 0$ and that the system is controllable, $P_{k} \in \mathbb{R}^{\left[n+m\left(p_{\mathrm{m}}+1\right)\right] \times\left[n+m\left(p_{\mathrm{m}}+1\right)\right]}$ is the symmetric positive-definite covariance matrix, $P_{\mathrm{x}, k} \triangleq P_{k} \boldsymbol{\zeta}_{k} \boldsymbol{\zeta}_{k}^{\top} P_{k}$ and $\mu_{k} \triangleq \zeta_{k}^{\top} P_{k} \zeta_{k}$.

\section{B. Control Law Design}

Taking the second order difference of the adaptive model (5),

$$
\begin{aligned}
& \hat{\mathbf{x}}_{k+1}-2 \hat{\mathbf{x}}_{k}+\hat{\mathbf{x}}_{k-1} \\
&=\hat{F}_{k} \mathbf{x}_{k}+\sum_{i=0}^{p_{m}} \hat{\Psi}_{i, k} \mathbf{u}_{k-i} \\
& \quad-2 \hat{F}_{k-1} \mathbf{x}_{k-1}-2 \sum_{i=0}^{p_{\mathrm{m}}} \hat{\Psi}_{i, k-1} \mathbf{u}_{k-i-1} \\
& \quad+\hat{F}_{k-2} \mathbf{x}_{k-2}+\sum_{i=0}^{p_{\mathrm{m}}} \hat{\Psi}_{i, k-2} \mathbf{u}_{k-i-2}
\end{aligned}
$$

Since $\hat{\mathbf{x}}_{k}=\tilde{\mathbf{x}}_{k}-\mathbf{x}_{k}$, this can be rearranged as

$$
\begin{aligned}
\hat{\mathbf{x}}_{k+1} & =\left(\hat{F}_{k}+2 I\right) \mathbf{x}_{k}-\left(2 \hat{F}_{k-1}+I\right) \mathbf{x}_{k-1}+\hat{F}_{k-2} \mathbf{x}_{k-2} \\
& +\sum_{i=0}^{p_{\mathrm{m}}}\left[\hat{\Psi}_{i, k} \mathbf{u}_{k-i}-2 \hat{\Psi}_{i, k-1} \mathbf{u}_{k-i-1}+\hat{\Psi}_{i, k-2} \mathbf{u}_{k-i-2}\right] \\
& -2 \tilde{\mathbf{x}}_{k}+\tilde{\mathbf{x}}_{k-1}
\end{aligned}
$$

which can be expressed as the equivalent augmented system

$$
\begin{aligned}
{\left[\begin{array}{c}
\hat{\mathbf{x}}_{k+1} \\
\mathbf{x}_{k} \\
\mathbf{x}_{k-1}
\end{array}\right]=} & {\left[\begin{array}{ccc}
\left(\hat{F}_{k}+2 I\right) & -\left(2 \hat{F}_{k-1}+I\right) & \hat{F}_{k-2} \\
I & {[0]} & {[0]} \\
{[0]} & I & {[0]}
\end{array}\right]\left[\begin{array}{c}
\mathbf{x}_{k} \\
\mathbf{x}_{k-1} \\
\mathbf{x}_{k-2}
\end{array}\right] } \\
& +\sum_{i=0}^{p_{\mathrm{m}}}\left[\begin{array}{ccc}
\hat{\Psi}_{i, k} & -2 \hat{\Psi}_{i, k-1} & \hat{\Psi}_{i, k-2} \\
{[0]} & {[0]} & {[0]} \\
{[0]} & {[0]} & {[0]}
\end{array}\right]\left[\begin{array}{c}
\mathbf{u}_{k-i} \\
\mathbf{u}_{k-i-1} \\
\mathbf{u}_{k-i-2}
\end{array}\right] \\
& +\left[\begin{array}{c}
\left.-2 \tilde{\mathbf{x}}_{k}+\tilde{\mathbf{x}}_{k-1}\right] \\
0 \\
0
\end{array}\right] \\
\triangleq & \hat{\Phi}_{k}\left[\begin{array}{c}
\mathbf{x}_{k} \\
\mathbf{x}_{k-1} \\
\mathbf{x}_{k-2}
\end{array}\right]+\sum_{i=0}^{p_{m}} \hat{\Gamma}_{i, k} \overline{\mathbf{u}}_{k-i}+\boldsymbol{\epsilon}_{k}
\end{aligned}
$$

where $\hat{\Phi}_{k} \in \mathbb{R}^{3 n \times 3 n}, \hat{\Gamma}_{i, k} \in \mathbb{R}^{3 n \times 3 m}, \overline{\mathbf{u}}_{k-i} \in \mathbb{R}^{3 m}$, and $\boldsymbol{\epsilon}_{k} \in \mathbb{R}^{3 n}$ have been introduced to simplify the notation.

In this form, it is now possible to introduce the substitutions used to transform the system into a delay-free system:

$$
\begin{gathered}
\hat{\boldsymbol{\eta}}_{k+1} \triangleq\left[\begin{array}{c}
\hat{\mathbf{x}}_{k+1} \\
\mathbf{x}_{k} \\
\mathbf{x}_{k-1}
\end{array}\right]+\sum_{i=1}^{p_{\mathrm{m}}} \hat{\Omega}_{i, k} \overline{\mathbf{u}}_{k-i+1} \\
\boldsymbol{\eta}_{k} \triangleq\left[\begin{array}{c}
\mathbf{x}_{k} \\
\mathbf{x}_{k-1} \\
\mathbf{x}_{k-2}
\end{array}\right]+\sum_{i=1}^{p_{\mathrm{m}}} \hat{\Omega}_{i, k} \overline{\mathbf{u}}_{k-i}
\end{gathered}
$$


where $\hat{\boldsymbol{\eta}}_{k+1}, \boldsymbol{\eta}_{k} \in \mathbb{R}^{3 n}$ and $\hat{\Omega}_{1, k}, \ldots, \hat{\Omega}_{p_{m}, k} \in \mathbb{R}^{3 n \times 3 m}$ are adaptive parameters whose derivation will be given shortly. The plant model can then be expressed as a system in terms of $\hat{\boldsymbol{\eta}}_{k+1}$ and $\boldsymbol{\eta}_{k}$. To do that, substitute (14) and (15) into the augmented plant (13), and add and subtract the term $\hat{\Omega}_{0, k} \overline{\mathbf{u}}_{k}$ :

$$
\begin{aligned}
\hat{\boldsymbol{\eta}}_{k+1}= & \hat{\Phi}_{k} \boldsymbol{\eta}_{k}+\hat{\Omega}_{0, k} \overline{\mathbf{u}}_{k}-\hat{\Phi}_{k} \sum_{i=1}^{p_{\mathrm{m}}} \hat{\Omega}_{i, k} \overline{\mathbf{u}}_{k-i}-\hat{\Omega}_{0, k} \overline{\mathbf{u}}_{k} \\
& +\sum_{i=0}^{p_{\mathrm{m}}} \hat{\Omega}_{i, k} \overline{\mathbf{u}}_{k-i}+\sum_{i=1}^{p_{\mathrm{m}}} \hat{\Omega}_{i, k} \overline{\mathbf{u}}_{k-i+1}+\boldsymbol{\epsilon}_{k} \\
= & \hat{\Phi}_{k} \boldsymbol{\eta}_{k}+\hat{\Omega}_{0, k} \overline{\mathbf{u}}_{k}-\left(\hat{\Omega}_{0, k}-\hat{\Omega}_{1, k}\right) \overline{\mathbf{u}}_{k}-\sum_{i=1}^{p_{\mathrm{m}}-1}\left(\hat{\Phi}_{k} \hat{\Omega}_{i, k}\right. \\
& \left.-\hat{\Omega}_{i+1, k}\right) \overline{\mathbf{u}}_{k-i}-\hat{\Phi}_{k} \hat{\Omega}_{p_{\mathrm{m}}, k} \overline{\mathbf{u}}_{k-p_{\mathrm{m}}}+\sum_{i=0}^{p_{\mathrm{m}}} \hat{\Gamma}_{i, k} \overline{\mathbf{u}}_{k-i}+\boldsymbol{\epsilon}_{k}
\end{aligned}
$$

The adaptive parameters $\hat{\Omega}_{0, k}, \ldots, \hat{\Omega}_{p_{m}, k}$ are to be computed from the parameter estimates produced by the adaptation laws (8) - (10), viz. $\hat{\Phi}_{k}$ and $\hat{\Gamma}_{0, k}, \ldots, \hat{\Gamma}_{p_{\mathrm{m}}, k}$, using the formula

$$
\hat{\Omega}_{i, k}= \begin{cases}\sum_{j=0}^{p_{\mathrm{m}}} \hat{\Phi}_{k}^{-j} \hat{\Gamma}_{j, k} & i=0 \\ \sum_{j=i}^{p_{\mathrm{m}}} \hat{\Phi}_{k}^{i-j-1} \hat{\Gamma}_{j, k} & i \in\left[1, p_{\mathrm{m}}\right]\end{cases}
$$

This reduces the dynamics in (16) to a delay-free system given by

$$
\hat{\boldsymbol{\eta}}_{k+1}=\hat{\Phi}_{k} \boldsymbol{\eta}_{k}+\hat{\Omega}_{0, k} \overline{\mathbf{u}}_{k}+\boldsymbol{\epsilon}_{k}
$$

Note that the difference between $\hat{\boldsymbol{\eta}}_{k+1}$ and $\boldsymbol{\eta}_{k+1}$ is

$$
\boldsymbol{\eta}_{k+1}-\hat{\boldsymbol{\eta}}_{k+1}=\left[\begin{array}{c}
\tilde{\mathbf{x}}_{k+1} \\
0 \\
0
\end{array}\right]+\sum_{i=1}^{p_{\mathrm{m}}} \Delta \hat{\Omega}_{i, k+1} \overline{\mathbf{u}}_{k-i+1}
$$

where $\Delta \hat{\Omega}_{i, k+1} \triangleq \hat{\Omega}_{i, k+1}-\hat{\Omega}_{i, k}$, so the delay-free system purely in terms of $\boldsymbol{\eta}_{k+1}$ is just

$$
\begin{aligned}
\boldsymbol{\eta}_{k+1} & =\hat{\Phi}_{k} \boldsymbol{\eta}_{k}+\hat{\Omega}_{0, k} \overline{\mathbf{u}}_{k}+\boldsymbol{\epsilon}_{k}+\left[\begin{array}{c}
\tilde{\mathbf{x}}_{k+1} \\
0 \\
0
\end{array}\right]+\sum_{i=1}^{p_{\mathrm{m}}} \Delta \hat{\Omega}_{i, k+1} \overline{\mathbf{u}}_{k-i+1} \\
& =\hat{\Phi}_{k} \boldsymbol{\eta}_{k}+\hat{\Omega}_{0, k} \overline{\mathbf{u}}_{k}+\sum_{i=1}^{p_{\mathrm{m}}} \Delta \hat{\Omega}_{i, k+1} \overline{\mathbf{u}}_{k-i+1}+\left[\begin{array}{c}
\Delta^{2} \tilde{\mathbf{x}}_{k+1} \\
0 \\
0
\end{array}\right]
\end{aligned}
$$

where $\Delta^{2} \tilde{\mathbf{x}}_{k+1} \triangleq \tilde{\mathbf{x}}_{k+1}-2 \tilde{\mathbf{x}}_{k}+\tilde{\mathbf{x}}_{k-1}$ is the second order difference of the model estimation error $\tilde{\mathbf{x}}_{k+1}$.

To make the system (20) internally stable, it is possible to use a control law of the form

$$
\overline{\mathbf{u}}_{k}=-L_{k}^{\top} \boldsymbol{\eta}_{k}
$$

where $L_{k} \in \mathbb{R}^{3 n \times 3 m}$ is the feedback gain matrix which can be found by pole-placement, provided that $\left(\hat{\Phi}_{k}, \hat{\Omega}_{0, k}\right)$ is a controllable pair. The controllability of the pair $\left(\hat{\Phi}_{k}, \hat{\Omega}_{0, k}\right)$ is addressed in Lemma 1.
Lemma 1: It is possible to select the initial adaptive law parameters and the coefficient $\alpha_{k}$ such that:

(a) The matrix $\hat{F}_{k}$ is non-singular, i.e., $\left|\hat{F}_{k}\right| \neq 0$.

(b) The pair $\bar{\Phi}_{k}, \hat{\Omega}_{0, k}$ is controllable.

Proof: To prove part (a) of Lemma 1, consider the adaptive law (8) for $k \in\left[k_{0}, \infty\right)$ and define a matrix

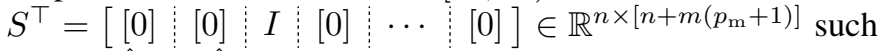
that $S^{\top} \hat{\Theta}_{k}=\hat{\Phi}_{3, k}$ and

$$
\begin{aligned}
S^{\top} \hat{\Theta}_{k+1} & =\hat{F}_{k+1} \\
& =\hat{F}_{k}+\alpha_{k} \rho_{k} S^{\top} P_{k+1} \boldsymbol{\zeta}_{k} \tilde{\mathbf{x}}_{k+1}^{\top} \\
& =\hat{F}_{k}\left[I+\alpha_{k} \rho_{k} \hat{F}_{k}^{-1} S^{\top} P_{k+1} \boldsymbol{\zeta}_{k} \tilde{\mathbf{x}}_{k+1}^{\top}\right]
\end{aligned}
$$

From (22), if the initial value $\left|\hat{F}_{k_{0}}\right| \neq 0$ and $\alpha_{k_{0}}^{-1}$ is not an eigenvalue of the matrix $-\hat{F}_{k_{0}}^{-1} S^{\top} P_{k_{0}+1} \boldsymbol{\zeta}_{k_{0}} \tilde{\mathbf{x}}_{k_{0}+1}^{\top}$ then $\hat{F}_{k_{0}+1}$ is non-singular. This can then be generalized for all $k \in\left[k_{0}, \infty\right)$ as a requirement that $\alpha_{k}^{-1}$ not be an eigenvalue of the matrix $-\hat{F}_{k}^{-1} S^{\top} P_{k+1} \zeta_{k} \tilde{\mathbf{x}}_{k+1}^{\top}$. This completes the proof of part (a) of Lemma 1.

To prove part (b) of Lemma 1, consider the pair $\bar{\Phi}_{k}, \hat{\Omega}_{0, k}$ and the fact that controllability requires that the controllability matrix $W_{\mathrm{c}, k} \triangleq\left[\begin{array}{l:l:l:l}\hat{\Omega}_{0, k} & \bar{\Phi}_{k} \hat{\Omega}_{0, k} & \cdots & \bar{\Phi}_{k}^{n-1} \hat{\Omega}_{0, k}\end{array}\right] \in$ $\mathbb{R}^{3 n \times 9 n \cdot m}$ be of rank $3 n$. To express $W_{\mathrm{c}, k}$ explicitly in terms of the adaptive parameters, $\hat{\Omega}_{0, k}$ in (17) is given as

$$
\hat{\Omega}_{0, k}=\hat{\Gamma}_{0, k}+\hat{\Phi}_{k}^{-1} \hat{\Gamma}_{1, k}+\cdots+\hat{\Phi}_{k}^{-p_{\mathrm{m}}} \bar{\Gamma}_{p_{\mathrm{m}}, k} .
$$

Substitution of (23) in the definition of the controllability matrix $W_{\mathrm{c}, k}$, it is obtained that

$$
W_{\mathrm{c}, k}=\sum_{i=0}^{p_{\mathrm{m}}} \hat{\Phi}_{k}^{-i}\left[\hat{\Gamma}_{i, k}: \hat{\Phi}_{k} \hat{\Gamma}_{i, k}: \cdots: \hat{\Phi}_{k}^{3 n-1} \hat{\Gamma}_{i, k}\right]
$$

which is now explicitly in terms of the adaptive parameters. Since (24) relies on the inverse of $\bar{\Phi}_{k}$ it is convenient to define $W_{\Phi, k} \triangleq \bar{\Phi}_{k}^{p_{\mathrm{m}}} W_{\mathrm{c}, k}$ such that the premultiplication of both sides of (24) with $\hat{\Phi}_{k}^{p_{\mathrm{m}}}$ results in

$$
W_{\Phi, k}=\sum_{i=0}^{p_{\mathrm{m}}} \bar{\Phi}_{k}^{p_{\mathrm{m}}-i}\left[\hat{\Gamma}_{i, k}: \hat{\Phi}_{k} \hat{\Gamma}_{i, k}: \cdots: \hat{\Phi}_{k}^{3 n-1} \hat{\Gamma}_{i, k}\right] .
$$

Consider now the adaptive law (8) when $\left|\hat{F}_{k}\right| \neq 0$. The adaptive law for each parameter can be written as

$$
\begin{array}{cc}
\hat{F}_{k+1} & =\hat{F}_{k}+\alpha_{k} \Lambda_{k} \\
\hat{\Psi}_{0, k+1} & =\hat{\Psi}_{0, k}+\alpha_{k} H_{0, k} \\
\vdots & \vdots \\
\hat{\Psi}_{p_{\mathrm{m}}, k+1} & =\hat{\Psi}_{p_{\mathrm{m}}, k}+\alpha_{k} H_{p_{\mathrm{m}}, k}
\end{array}
$$

where $\Lambda_{k}=\rho_{k} \tilde{\mathbf{x}}_{k+1} \boldsymbol{\zeta}_{k}^{\top} P_{k+1} C_{\phi}^{\top}$ and $H_{i, k}=$ $\rho_{k} \tilde{\mathbf{x}}_{k+1} \boldsymbol{\zeta}_{k}^{\top} P_{k+1} C_{\psi, i}^{\top} \forall i \in\left[0, p_{\mathrm{m}}\right]$ with $C_{\phi}$ being the 1 to $n$ rows of an identity matrix of size $n+m\left(p_{\mathrm{m}}+1\right)$ and 
$C_{\psi, i}$ being the $i \cdot m+n+1$ to $n+(i+1) m$ rows of an identity matrix of size $n+m\left(p_{\mathrm{m}}+1\right)$. Then $\hat{\Phi}_{k}$ is written as

$$
\begin{array}{r}
\hat{\Phi}_{k+1} \\
=\hat{\Phi}_{k}+\alpha_{k}\left[\begin{array}{ccc}
\Lambda_{k}+\frac{2}{\alpha_{k}} I & -\frac{1}{\alpha_{k}}\left(2 \alpha_{k-1} \Lambda_{k-1}+I\right) & \frac{\alpha_{k-2}}{\alpha_{k}} \Lambda_{k-2} \\
{[0]} & \ldots & {[0]} \\
\vdots & \ldots & \vdots \\
{[0]} & \ldots & {[0]}
\end{array}\right] \\
=\bar{\Phi}_{k}+\alpha_{k} \bar{\Lambda}_{k}
\end{array}
$$

and $\hat{\Gamma}_{i, k} \forall i \in\left[0, p_{\mathrm{m}}\right]$ is similarly written as

$$
\hat{\Gamma}_{i, k+1}=\hat{\Gamma}_{i, k}+\alpha_{k} \bar{H}_{i, k}
$$

Substitution of (27) and (28) in (25), results in an expression for $W_{\Phi, k}$ given as

$$
\begin{aligned}
W_{\Phi, k+1} & \sum_{i=0}^{p_{\mathrm{m}}}\left(\hat{\Phi}_{k}^{p_{\mathrm{m}}-i}+\alpha_{k} Q_{p_{\mathrm{m}}-i, k}\right)\left[\left(\hat{\Gamma}_{i, k}+\alpha_{k} \bar{H}_{i, k}\right)\right. \\
= & \left(\hat{\Phi}_{k}+\alpha_{k} Q_{1, k}\right)\left(\hat{\Gamma}_{i, k}+\alpha_{k} \bar{H}_{i, k}\right) \cdots\left(\hat{\Phi}_{k}^{3 n-1}\right. \\
& \left.\left.+\alpha_{k} Q_{3 n-1, k}\right)\left(\hat{\Gamma}_{i, k}+\alpha_{k} \bar{H}_{i, k}\right)\right] \\
= & \sum_{i=0}^{p_{\mathrm{m}}} \hat{\Phi}_{k}^{p_{\mathrm{m}}-i}\left[\hat{\Gamma}_{i, k}: \hat{\Phi}_{k} \hat{\Gamma}_{i, k} \ldots \hat{\Phi}_{k}^{3 n-1} \hat{\Gamma}_{i, k}\right] \\
& +\alpha_{k} \sum_{i=0}^{p_{\mathrm{m}}}\left[\hat{\Phi}_{k}^{p_{\mathrm{m}}-i} \bar{H}_{i, k}+Q_{p_{\mathrm{m}}-i, k} \hat{\Gamma}_{i, k+1} \hat{\Phi}_{k} \bar{H}_{i, k}\right. \\
& +Q_{1, k} \hat{\Gamma}_{i, k+1}+Q_{p_{\mathrm{m}}-i, k} \hat{\Phi}_{k+1} \hat{\Gamma}_{i, k+1}{ }^{\cdots} \hat{\Phi}_{k}^{3 n-1} \\
& \left.\times \bar{H}_{i, k}+Q_{3 n-1, k} \hat{\Gamma}_{i, k+1}+Q_{p_{\mathrm{m}}-i, k} \hat{\Phi}_{k}^{3 n-1} \hat{\Gamma}_{i, k+1}\right]
\end{aligned}
$$

where $Q_{i, k} \triangleq \alpha_{k}^{-1}\left(\hat{\Phi}_{k+1}^{i}-\hat{\Phi}_{k}^{i}\right)$. Note that the first term on the right-hand-side of (29) is a single time-step delayed (25). Therefore, (29) is simplified as

$$
W_{\Phi, k+1}=W_{\Phi, k}+\alpha_{k} M_{k+1}
$$

where

$$
\begin{aligned}
M_{k+1}= & \sum_{i=0}^{p_{\mathrm{m}}}\left[\hat{\Phi}_{k}^{p_{\mathrm{m}}-i} \bar{H}_{i, k}+Q_{p_{\mathrm{m}}-i, k} \hat{\Gamma}_{i, k+1}\right. \\
& \times \hat{\Gamma}_{i, k+1}+\hat{\Phi}_{k} \bar{H}_{i, k}+Q_{1, k} \\
& \left.+Q_{3 n-1, k} \hat{\Gamma}_{i, k+1}+\hat{\Phi}_{k+1} \hat{\Gamma}_{i, k+1} \cdots \hat{\Phi}_{p_{\mathrm{m}}-i, k}^{3 n-1} \hat{\Phi}_{k}^{3 n-1} \hat{\Gamma}_{i, k+1}\right]
\end{aligned}
$$

Consider now the expression (30) when $k=k_{0}+1$ and suppose that the initial adaptive parameters are selected such that $W_{\Phi, k_{0}}$ has a rank of $3 n$ which implies $W_{\Phi, k_{0}} W_{\Phi, k_{0}}^{\top}$ is a non-singular matrix then it is obtained that

$$
\begin{aligned}
W_{\Phi, k_{0}+1} & W_{\Phi, k_{0}+1}^{\top} \\
= & W_{\Phi, k_{0}} W_{\Phi, k_{0}}^{\top}\left(I+\alpha_{k_{0}}\left(W_{\Phi, k_{0}} W_{\Phi, k_{0}}^{\top}\right)^{-1}\left(W_{\Phi, k_{0}}\right.\right. \\
& \left.\left.\times M_{k_{0}+1}^{\top}+W_{\Phi, k_{0}}^{\top} M_{k_{0}+1}+\alpha_{k} M_{k_{0}+1} M_{k_{0}+1}^{\top}\right)\right)
\end{aligned}
$$

where $W_{\Phi, k_{0}+1}$ has a rank of $3 n$ if and only if $\alpha_{k_{0}}^{-1} \neq \lambda\left[-\left(W_{\Phi, k_{0}} W_{\Phi, k_{0}}^{\top}\right)^{-1}\left(W_{\Phi, k_{0}} M_{k_{0}+1}^{\top}+W_{\Phi, k_{0}}^{\top} M_{k_{0}+1}\right.\right.$ $\left.\left.+\alpha_{k_{0}} M_{k_{0}+1} M_{k_{0}+1}^{\top}\right)\right]$, where $\lambda[\cdot]$ is the set of eigenvalues. Then, in general, $W_{\Phi, k}$ has a rank of $3 n$ if the initial value $W_{\Phi, k_{0}}$ is also has a rank of $3 n$ and $\alpha_{k}^{-1} \neq \lambda\left[-\left(W_{\Phi, k} W_{\Phi, k}^{\top}\right)^{-1}\left(W_{\Phi, k} M_{k+1}^{\top}+W_{\Phi, k}^{\top} M_{k+1}\right.\right.$ $\left.\left.+\alpha_{k} M_{k+1} M_{k+1}^{\top}\right)\right]$.

Furthermore, since $\hat{\Phi}_{k}$ is a non-singular matrix, if $W_{\Phi, k}$ has a rank of $3 n$ then $W_{\mathrm{c}, k}$ also has a rank of $3 n$ and the pair $\bar{\Phi}_{k}, \hat{\Omega}_{0, k}$ is controllable.

Remark 1: Similar to in [8], the coefficient $\alpha_{k}>0$ can be selected from a set of pre-defined values to ensure that $W_{\mathrm{c}, k}$ has a rank of $3 n$.

\section{Stability Analysis}

In this section, it is shown that the parameter adaptation produces bounded and convergent parameter estimates (Lemma 2 and Lemma 3), that the adaptive system model converges in input-output behaviour to the true system (Lemma 4), and that the proposed adaptive control law drives the system state to zero asymptotically (Theorem $\mathbf{1}$ ).

Lemma 2: For the system (4) with the adaptive laws (8) and (9) it is true that

$$
\lim _{k \rightarrow \infty} \frac{\alpha_{k} \rho_{k}}{1+\alpha_{k} \rho_{k} \mu_{k}} \tilde{\mathbf{x}}_{k}^{\top} \tilde{\mathbf{x}}_{k}=0
$$

Furthermore, it is also true that the parameter estimate $\hat{\boldsymbol{\theta}}_{k}$ is bounded, hence, the parameter estimation error $\tilde{\boldsymbol{\theta}}_{k}$ is also bounded.

Proof: To proceed with the proof, let $\tilde{\mathbf{x}}_{k}^{\top}=$ $\left[\begin{array}{l:l:l}\tilde{x}_{1, k} & \cdots & \tilde{x}_{n, k}\end{array}\right]^{\top}, \tilde{\Theta}_{k}^{\top}=\left[\begin{array}{lll:l}\tilde{\boldsymbol{\theta}}_{1, k} & \cdots & \tilde{\boldsymbol{\theta}}_{n, k}\end{array}\right]^{\top}$ and consider the following positive function

$$
V_{k}=\sum_{j=1}^{n} \tilde{\boldsymbol{\theta}}_{j, k}^{\top} P_{k}^{-1} \tilde{\boldsymbol{\theta}}_{j, k} .
$$

The forward difference of (34) is given by

$$
\begin{aligned}
\Delta V_{k} & =V_{k+1}-V_{k} \\
& =\sum_{j=1}^{m}\left[\tilde{\boldsymbol{\theta}}_{j, k+1}^{\top} P_{k+1}^{-1} \tilde{\boldsymbol{\theta}}_{j, k+1}-\tilde{\boldsymbol{\theta}}_{j, k}^{\top} P_{k}^{-1} \tilde{\boldsymbol{\theta}}_{j, k}\right] .
\end{aligned}
$$

Following an approach similar to in [8] it is obtained that

$$
\lim _{k \rightarrow \infty} \Delta V_{k}=\lim _{k \rightarrow \infty} \frac{\alpha_{k} \rho_{k}}{1+\alpha_{k} \rho_{k} \mu_{k}} \tilde{\mathbf{x}}_{k}^{\top} \tilde{\mathbf{x}}_{k}=0
$$


which is true for $\left\|\tilde{\mathbf{x}}_{k}\right\| \geq \omega_{\max }$, [8]. The result (36) implies that $\lim _{k \rightarrow \infty}\left\|\tilde{\Theta}_{k+1}-\tilde{\Theta}_{k}\right\|=0$. Consequently, $\lim _{k \rightarrow \infty}\left\|\hat{\Theta}_{k+1}-\hat{\Theta}_{k}\right\|=0,[8]$.

Lemma 3: Using the results in Lemma 2, the vector $\boldsymbol{\eta}_{k}$ defined in (14) is bounded as

$$
\left\|\boldsymbol{\eta}_{k}\right\| \leq c_{0}+c_{1} \max _{i \in[0, k]}\left\|\tilde{\mathbf{x}}_{k-i}\right\|
$$

for some positive constants $c_{0}, c_{1}$ and, consequently, the model estimation error $\tilde{\mathbf{x}}_{k}$ converges to a bound of $\omega_{\max }$ asymptotically, i.e.

$$
\lim _{k \rightarrow \infty}\left\|\tilde{\mathbf{x}}_{k}\right\| \leq \omega_{\max }
$$

Proof: Consider (14), the difference of the two vectors results in the expression

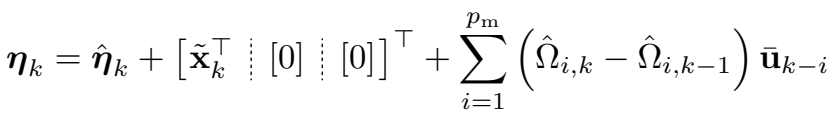

$$
\begin{aligned}
& =\hat{\boldsymbol{\eta}}_{k}+\left[\begin{array}{l:l:l}
\tilde{\mathbf{x}}_{k}^{\top} & {[0]} & {[0]}
\end{array}\right]^{\top}+\sum_{i=1}^{p_{\mathrm{m}}} \Delta \hat{\Omega}_{i, k} \overline{\mathbf{u}}_{k-i}
\end{aligned}
$$

where $\Delta \hat{\Omega}_{i, k} \triangleq \hat{\Omega}_{i, k}-\hat{\Omega}_{i, k-1}$. Substitution of (20) and (21) in a one time-step forward (39)

$$
\begin{aligned}
\boldsymbol{\eta}_{k+1}= & \hat{\Phi}_{\mathrm{m}, k} \boldsymbol{\eta}_{k}+\sum_{i=1}^{p_{\mathrm{m}}} \Delta \hat{\Omega}_{i, k} \overline{\mathbf{u}}_{k-i}+\left[\Delta^{2} \tilde{\mathbf{x}}_{k+1}^{\top}:[0]:[0]\right]^{\top} \\
= & \hat{\Phi}_{\mathrm{m}, k} \boldsymbol{\eta}_{k}-\sum_{i=1}^{p_{\mathrm{m}}} \Delta \hat{\Omega}_{i, k} L_{k-i}^{\top} \boldsymbol{\eta}_{k-i}+\left[\Delta^{2} \tilde{\mathbf{x}}_{k+1}^{\top}:[0]\right. \\
& {[0]]^{\top} }
\end{aligned}
$$

where $\hat{\Phi}_{\mathrm{m}, k}=\hat{\Phi}_{k}-\hat{\Omega}_{0, k} L_{k}^{\top}$. Expressing (40) in augmented form and defining $N_{i, k} \triangleq \Delta \hat{\Omega}_{i, k} L_{k-i}^{\top} \in \mathbb{R}^{3 n \times 3 n}$ such that,

$$
\begin{aligned}
& \overline{\boldsymbol{\eta}}_{k+1}=\left[\begin{array}{cccc}
\bar{\Phi}_{\mathrm{m}, k}-N_{1, k} & -N_{2, k} & \cdots & -N_{p_{\mathrm{m}}, k} \\
I & {[0]} & \cdots & {[0]} \\
{[0]} & I & \cdots & \vdots \\
\vdots & \vdots & \ddots & {[0]}
\end{array}\right] \overline{\boldsymbol{\eta}}_{k} \\
& +\left[\begin{array}{l|l:l:l}
\Delta^{2} \tilde{\mathbf{x}}_{k+1}^{\top} & 0 & \cdots & 0
\end{array}\right]^{\top}
\end{aligned}
$$

where $\overline{\boldsymbol{\eta}}_{k-1}^{\top} \triangleq\left[\begin{array}{l|l|l|l}\boldsymbol{\eta}_{k-1}^{\top} & \boldsymbol{\eta}_{k-2}^{\top} & \cdots & \boldsymbol{\eta}_{k-p_{\mathrm{m}}}^{\top}\end{array}\right] \in \mathbb{R}^{3 n \cdot p_{\mathrm{m}}}$. Using the results in Lemma 2 and [8], $\lim _{k \rightarrow \infty}\left\|N_{i, k-1}\right\|=0$ and that implies that the augmented system, (41), can be reduced to the form

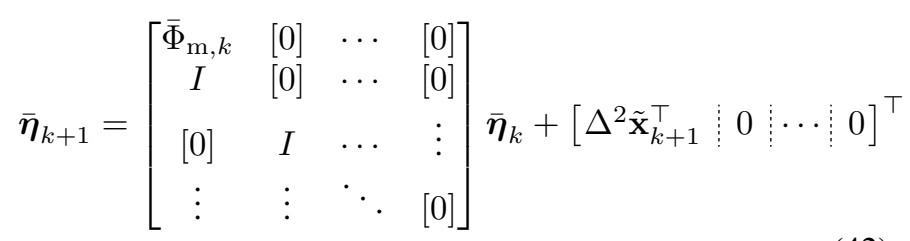

which is stable and has $3 n$ eigenvalues of the matrix $\hat{\Phi}_{\mathrm{m}, k}$ while the remaining $3 n \cdot p_{\mathrm{m}}-3 n$ eigenvalues are 0 . Therefore, the system (42) is stable and a bound on $\boldsymbol{\eta}_{k}$ exists such that

$$
\left\|\boldsymbol{\eta}_{k}\right\| \leq c_{0}+c_{1} \max _{i \in[0, k]}\left\|\Delta^{2} \tilde{\mathbf{x}}_{k-i}\right\|
$$

for some positive constants $c_{0}$ and $c_{1}$. This establishes the bound on $\boldsymbol{\eta}_{k}$.

Consider now the control law (21). From (43) and the fact that $L_{\mathrm{x}, k}$, is bounded then the control input is bounded as

$$
\left\|\mathbf{u}_{k}\right\| \leq c_{2}+c_{3} \max _{i \in[0, k]}\left\|\Delta^{2} \tilde{\mathbf{x}}_{k-i}\right\|
$$

for some positive constants $c_{2}$ and $c_{3}$. Using (39) and the fact that $\mathbf{x}_{k}=\hat{\mathbf{x}}_{k}+\tilde{\mathbf{x}}_{k}$ a bound on $\mathbf{x}_{k}$ is obtained as

$$
\left\|\mathbf{x}_{k}\right\| \leq c_{4}+c_{5} \max _{i \in[0, k]}\left\|\tilde{\mathbf{x}}_{k-i}\right\|
$$

for some positive constants $c_{4}$ and $c_{5}$. From the definition of $\zeta_{k}$ and using (44), (45) there exists positive constants $c_{0}^{0}$ and $c_{1}^{0}$ such that

$$
\left\|\boldsymbol{\zeta}_{k}\right\| \leq c_{0}^{0}+c_{1}^{0} \max _{i \in[0, k]}\left\|\tilde{\mathbf{x}}_{k-i}\right\| .
$$

Consequently, from (46) and the Key Technical Lemma, it is obtained that

$$
\lim _{k \rightarrow \infty}\left\|\tilde{\mathbf{x}}_{k}\right\| \leq \omega_{\max }
$$

Remark 2: Since $\left\|\tilde{\mathbf{x}}_{k}\right\|$ is uniformly bounded then, from (43), $\left\|\boldsymbol{\eta}_{k}\right\|$ is uniformly bounded. Furthermore, from (14) and the fact that the adaptive parameters are bounded then $\left\|\hat{\boldsymbol{\eta}}_{k}\right\|$ is also uniformly bounded.

Theorem 1: The states of the closed-loop system approaches a bound of $\epsilon \in O\left(T^{2}\right)$ around zero, i.e. $\lim _{k \rightarrow \infty}\left\|\mathbf{x}_{k}\right\| \leq \epsilon$.

Proof: Consider Lemma 3 and the stable dynamics given by (42), which is reduced to the form

$$
\boldsymbol{\eta}_{k+1}=\hat{\Phi}_{\mathrm{m}, k} \boldsymbol{\eta}_{k}+\left[\begin{array}{l:lll:l}
\Delta^{2} \tilde{\mathbf{x}}_{k}^{\top} & 0 & \cdots & 0
\end{array}\right]^{\top} .
$$

From Lemma 2, it is shown that the adaptive parameters are bounded and converge at steady state. Therefore, there exists $\hat{\Phi}_{\mathrm{m}, \mathrm{ss}}$ and $\hat{\Omega}_{0, \mathrm{ss}}$ such that $\hat{\Phi}_{\mathrm{m}, \mathrm{ss}}=\lim _{k \rightarrow \infty} \hat{\Phi}_{\mathrm{m}, k}$ and $\hat{\Omega}_{0, \mathrm{ss}}=$ $\lim _{k \rightarrow \infty} \hat{\Omega}_{0, k}$. Then the dynamics (48) is written as

$$
\boldsymbol{\eta}_{k+1}=\hat{\Phi}_{\mathrm{m}, \mathrm{ss}} \boldsymbol{\eta}_{k}+\gamma_{k}
$$

where

$$
\boldsymbol{\gamma}_{k}=\left(\hat{\Phi}_{\mathrm{m}, k}-\hat{\Phi}_{\mathrm{m}, \mathrm{ss}}\right) \boldsymbol{\eta}_{k}+\left[\begin{array}{ll:l:l:l}
\Delta^{2} \tilde{\mathbf{x}}_{k}^{\top} & 0 & \cdots & 0
\end{array}\right]^{\top}
$$

and since all the terms on the right-hand-side of (50) are bounded then $\lim _{k \rightarrow \infty}\left\|\gamma_{k}\right\| \in O\left(\left\|\Delta^{2} \tilde{\mathbf{x}}_{k}\right\|\right)$. The solution of (49) is given as

$$
\boldsymbol{\eta}_{k}=\hat{\Phi}_{\mathrm{m}, \mathrm{ss}}^{k-k_{0}} \boldsymbol{\eta}_{k_{0}}+\sum_{i=k_{0}}^{k-1} \bar{\Phi}_{\mathrm{m}, \mathrm{ss}}^{i} \gamma_{k-i}
$$


where $\boldsymbol{\eta}_{k_{0}}$ is the initial value of the vector $\boldsymbol{\eta}_{k}$. At steady state $\lim _{k \rightarrow \infty} \boldsymbol{\eta}_{k}$ is given as

$$
\lim _{k \rightarrow \infty} \boldsymbol{\eta}_{k}=\bar{\gamma}_{k}
$$

where $\bar{\gamma}_{k}=\lim _{k \rightarrow \infty} \sum_{i=0}^{k-1} \bar{\Phi}_{\mathrm{m}, \mathrm{ss}}^{i} \gamma_{k-i} \in O\left(T^{-1}\right)$. $O\left(\left\|\Delta^{2} \tilde{\mathbf{x}}_{k}\right\|\right)$, [9]. Now, consider the definition of $\boldsymbol{\eta}_{k}$ given as

$$
\boldsymbol{\eta}_{k}=\left[\begin{array}{l:l:l}
\mathbf{x}_{k}^{\top} & \cdots & \mathbf{x}_{k-2}^{\top}
\end{array}\right]^{\top}+\sum_{i=0}^{p_{\mathrm{m}}} \hat{\Omega}_{i, k} \mathbf{u}_{k-i}
$$

premultiplying (53) with $C^{\top}=\left[\begin{array}{l:l:l:l}I & {[0]} & \cdots & {[0]}\end{array}\right] \in \mathbb{R}^{n \times 3 n}$ gives

$$
\mathbf{x}_{k}=C^{\top}\left(\boldsymbol{\eta}_{k}-\sum_{i=1}^{p_{\mathrm{m}}} \hat{\Omega}_{i, k} \mathbf{u}_{k-i}\right) .
$$

The steady state value $\lim _{k \rightarrow \infty} \mathbf{x}_{k}$ is given as

$$
\begin{aligned}
\lim _{k \rightarrow \infty} \mathbf{x}_{k} & =C^{\top} \lim _{k \rightarrow \infty}\left(\boldsymbol{\eta}_{k}-\sum_{i=1}^{p_{\mathrm{m}}} \hat{\Omega}_{i, k} \mathbf{u}_{k-i}\right) \\
& =C^{\top}\left(\lim _{k \rightarrow \infty} \boldsymbol{\eta}_{k}-\sum_{i=1}^{p_{\mathrm{m}}} \hat{\Omega}_{i, \mathrm{ss}} \lim _{k \rightarrow \infty} \mathbf{u}_{k-i}\right) .
\end{aligned}
$$

From (21) it is obtained that

$$
\lim _{k \rightarrow \infty} \mathbf{u}_{k}=-L_{s s}^{\top} \lim _{k \rightarrow \infty} \boldsymbol{\eta}_{k}
$$

If $\left\|L_{s s}\right\| \in O(1)$ and, since, $\lim _{k \rightarrow \infty}\left\|\boldsymbol{\eta}_{k}\right\| \in O\left(T^{-1}\right)$. $O\left(\left\|\Delta^{2} \tilde{\mathbf{x}}_{k}\right\|\right)$ then $\lim _{k \rightarrow \infty}\left\|\mathbf{u}_{k}\right\| \in O\left(T^{-1}\right) \cdot O\left(\left\|\Delta^{2} \tilde{\mathbf{x}}_{k}\right\|\right)$. Furthermore, since $\left\|\tilde{\mathbf{x}}_{k}\right\| \in\left\|\boldsymbol{\omega}_{k}\right\| \in O(T)$, then $O\left(\left\|\Delta^{2} \tilde{\mathbf{x}}_{k}\right\|\right) \in O\left(\left\|\Delta^{2} \boldsymbol{\omega}_{k}\right\|\right) \in O\left(T^{3}\right)$. Finally, from (55) and using the bounds on $\boldsymbol{\eta}_{k}$ and $\mathbf{u}_{k}$ while assumung that $\left\|\hat{\Omega}_{i, \mathrm{ss}}\right\| \in O(1)$ it is obtained that

$$
\lim _{k \rightarrow \infty}\left\|\mathbf{x}_{k}\right\| \leq \epsilon
$$

where $\epsilon \in O\left(T^{2}\right)+O\left(p_{\mathrm{m}}\right) \cdot O\left(T^{2}\right)$ and if the sampling interval $T$ is selected in such a way that $p_{\mathrm{m}} \in O(1)$ then $\epsilon \in O\left(T^{2}\right)$.

\section{SIMULATION EXAMPLE}

Consider an unstable $2^{\text {nd }}$-order continuous-time plant with time-delay given by

$$
\begin{aligned}
\dot{\mathbf{x}}(t)= & {\left[\begin{array}{ccc}
0 & 1 & 0 \\
0 & 0 & 1 \\
0.1 & -0.8 & -1.9
\end{array}\right] \mathbf{x}(t)+\left[\begin{array}{ll}
0 & 0 \\
1 & 0 \\
1 & 1
\end{array}\right]\left[\begin{array}{l}
u_{1}\left(t-\tau_{1}\right) \\
u_{2}\left(t-\tau_{2}\right)
\end{array}\right] } \\
& +\left[\begin{array}{l}
1 \\
1 \\
1
\end{array}\right] \sin (3 \pi t)
\end{aligned}
$$

where two cases are considered: $\tau_{1}=\tau_{2}=0$ s (i.e. no delay) and $\tau_{1}=0.1, \tau_{2}=0.3 \mathrm{~s}$ with $\tau_{\mathrm{p}}=0.5 \mathrm{~s}$. The sampling interval is selected as $T=0.1 \mathrm{~s}$ which, for each of the two different time-delays, results in the discrete-time plants respectively given by

$$
\begin{aligned}
\mathbf{x}_{k+1}= & {\left[\begin{array}{ccc}
1 & 0.0999 & 0.0047 \\
0.0005 & 0.9963 & 0.0910 \\
0.0091 & -0.0723 & 0.8234
\end{array}\right] \mathbf{x}_{k}+\left[\begin{array}{ll}
0.0052 & 0.0002 \\
0.1046 & 0.0047 \\
0.0872 & 0.0910
\end{array}\right] \mathbf{u}_{k} } \\
& +\boldsymbol{\omega}_{k}
\end{aligned}
$$

and

$$
\begin{aligned}
\mathbf{x}_{k+1}= & {\left[\begin{array}{ccc}
1 & 0.0999 & 0.0047 \\
0.0005 & 0.9963 & 0.0910 \\
0.0091 & -0.0723 & 0.8234
\end{array}\right] \mathbf{x}_{k}+\left[\begin{array}{cc}
0.0052 & 0 \\
0.1046 & 0 \\
0.0872 & 0
\end{array}\right] \mathbf{u}_{k-d_{1}} } \\
& +\left[\begin{array}{ll}
0 & 0.0002 \\
0 & 0.0047 \\
0 & 0.0910
\end{array}\right] \mathbf{u}_{k-d_{2}}+\boldsymbol{\omega}_{k}
\end{aligned}
$$

where $d_{1}=1, d_{2}=3$ and $p=5$, respectively. The initial condition of the plant is set at $\mathbf{x}(0)=\left[\begin{array}{lll}1 & -1 & 1\end{array}\right]^{\top}$.

To investigate the adaptive performance of the regulator under no delay conditions, the closed-loop system is simulated using an uncertainty of $20 \%$ on the paramters of (59). The adaptive regulator is initialised with $P_{0}=10^{2} \times I_{p+3 \times p+3}$. In Fig. 1-2 the results are shown for the state regulation of $\mathbf{x}(t)$ and the control input profile of the closed-loop plant under a delay upper-bound $\tau_{\mathrm{p}}=0 \mathrm{~s}$, for $\tau_{1}=\tau_{2}=0 \mathrm{~s}$ respectively. As expected, $x_{1}(t)$ is regulated to a bound of $O\left(0.1^{2}\right)$ asymptotically. Finally, the system is simulated for a delay of $\tau_{1}=0.1 \mathrm{~s}$ and $\tau_{2}=0.3 \mathrm{~s}$ with an upper-bound of $\tau_{\mathrm{p}}=0.5 \mathrm{~s}$. In Fig. 3-5 the results are shown for the state regulation of $x_{1}$, the control input profile and the elements of the matrix $\hat{F}$ of the closed-loop plant respectively. As expected, the adaptive parameters converge to constant values at steady state while the state $\mathbf{x}(t)$ is regulated to a bound of $O\left(0.1^{2}\right)$.

\section{CONCLUSIONS}

In this paper, a discrete-time adaptive regulator is proposed for a MIMO linear plant which is subject to an unmeasurable disturbance and has uncertain input delays that may differ across input channels. An adaptive plant model incorporating a second-order disturbance observer is formulated which does not depend on knowledge of the time delays, only their upper bound. The adaptation laws based on recursive least squares incorporate a dead zone that ensures stability in the presence of disturbance. To facilitate control law design, state substitutes are used to transform the plant into a delay-free dynamics, which was shown to be controllable. It was further shown that the proposed adaptive regulator drives the plant state to zero asymptotically, within an $O\left(T^{2}\right)$ bound. Simulation results demonstrate the ability of the adaptive regulator to handle a delay-free plant, as well as mismatches between the delay upper-bound and the true time delay. 


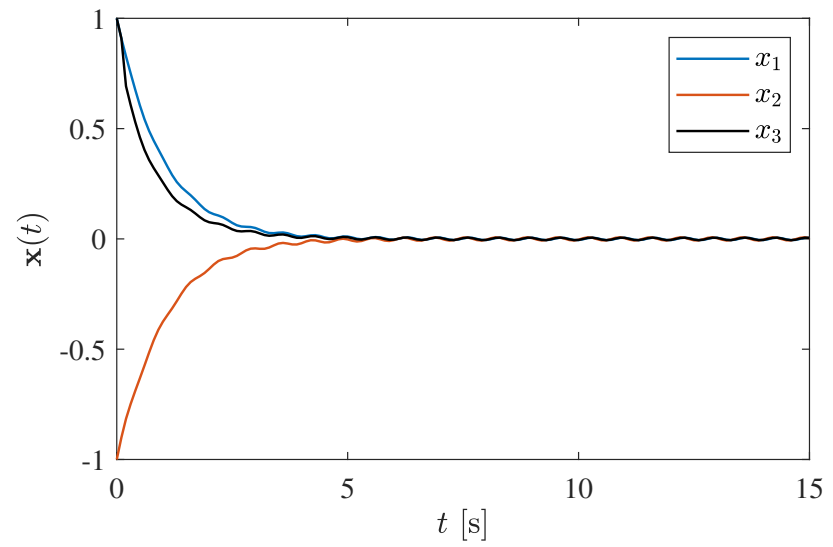

Fig. 1. State regulation of $x_{1}$ on the delay-free plant $\left(\tau_{1}=\tau_{2}=0 \mathrm{~s}\right)$, using a delay upper-bound $\tau_{\mathrm{p}}=0 \mathrm{~s}$.

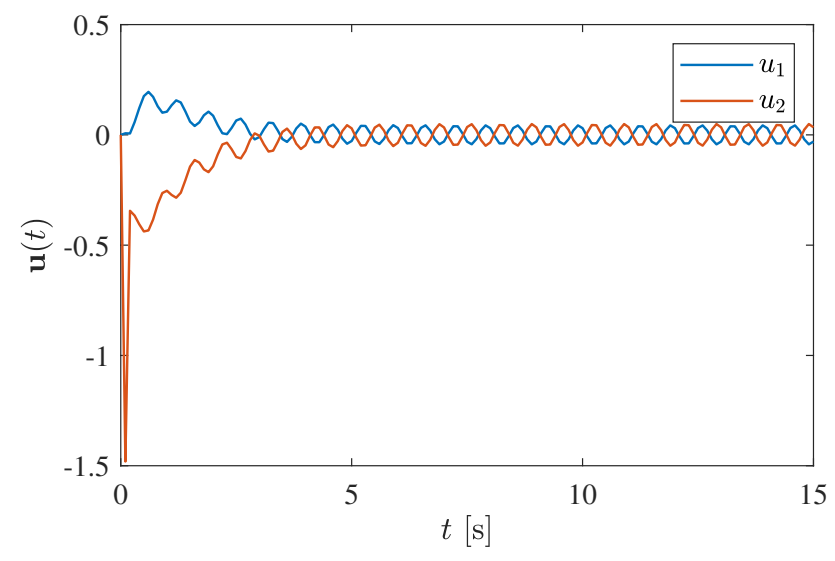

Fig. 2. Control input profile on the delay-free plant $\left(\tau_{1}=\tau_{2}=0\right.$ s), using a delay upper-bound $\tau_{\mathrm{p}}=0 \mathrm{~s}$.

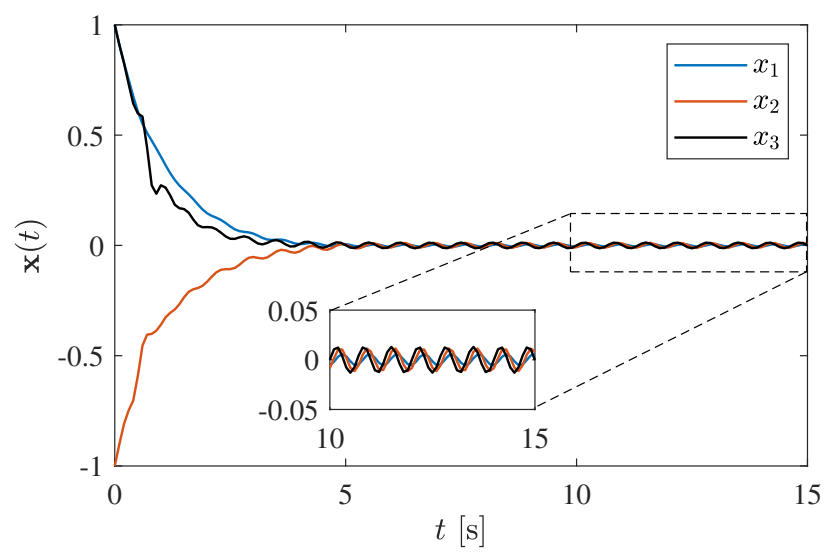

Fig. 3. State regulation $x_{1}$ on the plant with delay $\left(\tau_{1}=0.1 \mathrm{~s}\right.$ and $\left.\tau_{2}=0.3 \mathrm{~s}\right)$, using a delay upper-bound $\tau_{\mathrm{p}}=0.4 \mathrm{~s}$.

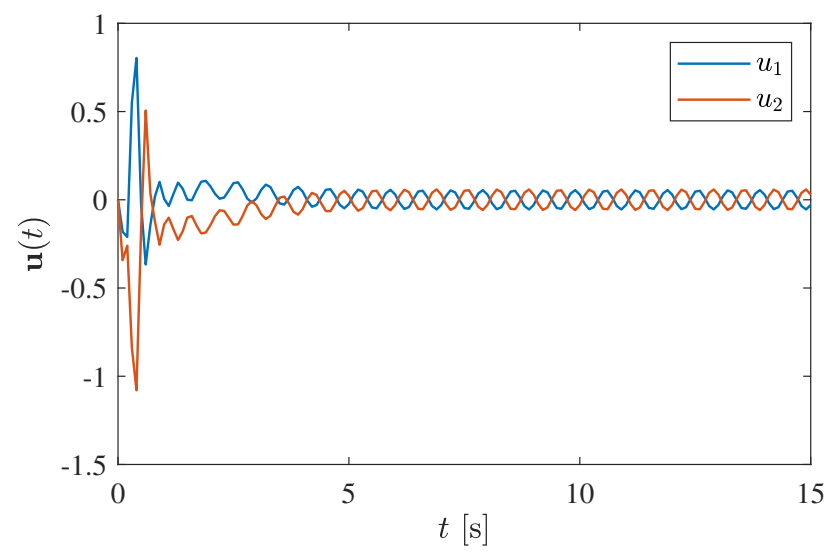

Fig. 4. Control input profile on the plant with delay $\left(\tau_{1}=0.1 \mathrm{~s}\right.$ and $\tau_{2}=$ $0.3 \mathrm{~s}$ ), using a delay upper-bound $\tau_{\mathrm{p}}=0.4 \mathrm{~s}$.

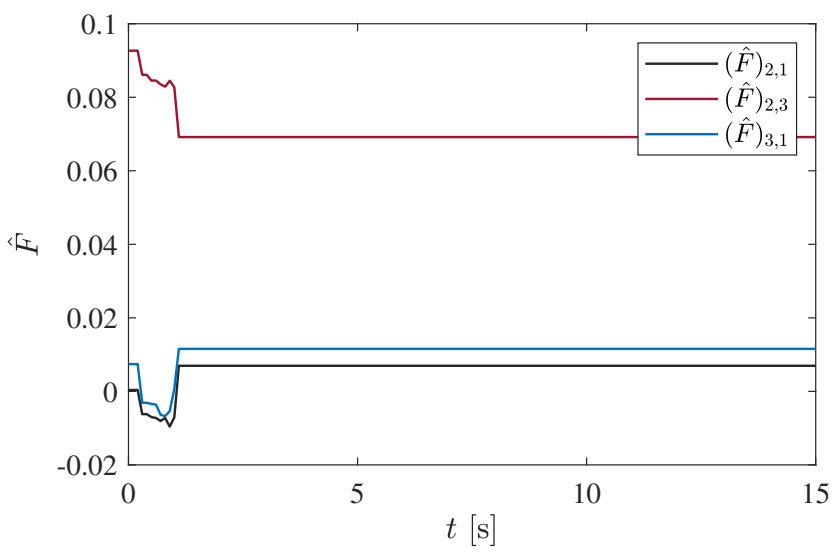

Fig. 5. Adaptive parameters $\hat{F}$ of the plant with delay $\left(\tau_{1}=0.1 \mathrm{~s}\right.$ and $\left.\tau_{2}=0.3 \mathrm{~s}\right)$, using a delay upper-bound $\tau_{\mathrm{p}}=0.4 \mathrm{~s}$.

\section{ACKNOWLEDGMENT \\ REFERENCES \\ REFERENCES}

[1] E. Fridman, Introduction to Time-Delay Systems: Analysis and Control, Birkhäuser, 2014.

[2] K. Gu, V. L. Kharitonov, and J. Chen, Stability of Time-Delay Systems, Birkhäuser, 2003.

[3] D. Ma and J. Chen, "Delay Margin of Low-Order Systems Achievable by PID Controllers," in IEEE Transactions on Automatic Control, vol. 64, no. 5, pp. 1958-1973, May 2019.

[4] T. Liu, S. Dong, F. Chen, and D. Li, "Identification of Discrete-Time Output Error Model Using Filtered Input Excitation for Integrating Processes With Time Delay," in IEEE Transactions on Automatic Control, vol. 62, no. 5, pp. 2524-2530, May 2017.

[5] Z. Artstein, "Linear systems with delayed controls: a reduction," IEEE Transactions on Automatic Control, vol. 27, no. 4, pp. 869-879, 1982.

[6] A. Gonzalez, "Robust stabilization of linear discrete-time systems with time-varying input delay," Automatica, vol. 49, no. 9, pp. 2919-2922, 2013.

[7] K. Abidi and H. J. Soo, "Discrete-time Adaptive Regulation of Systems with Uncertain Upper-bounded Input Delay: A State Substitution Approach," Proceedings of the 16th International Conference on Informatics in Control, Automation and Robotics, 27-30 April, 2019, Prague, Czech Republic. 
[8] K. Abidi, H. J. Soo, and I. Postlethwaite, "Discrete-Time Adaptive Control of Uncertain Sampled-Data Systems with Uncertain Input Delay: A Reduction," IET Control Theory and Applications, in press.

[9] K. Abidi, J. Xu, and X. Yu, "On the discrete-time integral sliding-mode control," IEEE Transactions on Automatic Control, vol. 52, no. 4, pp. 709-715, April 2007. 